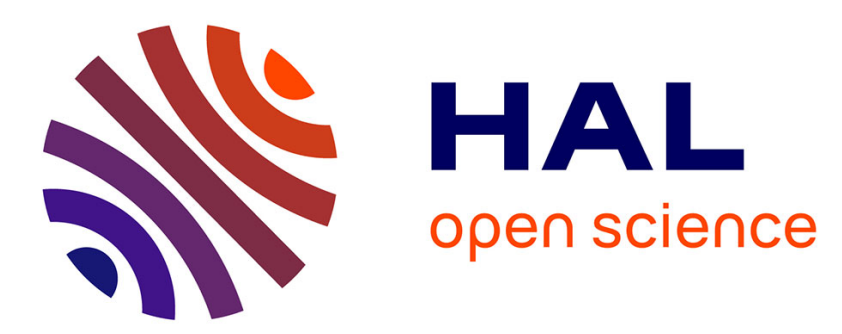

\title{
High-Energy Long-Lived Excited States in DNA Double Strands
}

\author{
I. Vaya, F.-A. Miannay, T. Gustavsson, D. Markovitsi
}

\section{To cite this version:}

I. Vaya, F.-A. Miannay, T. Gustavsson, D. Markovitsi. High-Energy Long-Lived Excited States in DNA Double Strands. ChemPhysChem, 2010, 11, pp.987 - 989. 10.1002/cphc.201000027 . hal00472807

\section{HAL Id: hal-00472807 https://hal.science/hal-00472807}

Submitted on 13 Apr 2010

HAL is a multi-disciplinary open access archive for the deposit and dissemination of scientific research documents, whether they are published or not. The documents may come from teaching and research institutions in France or abroad, or from public or private research centers.
L'archive ouverte pluridisciplinaire HAL, est destinée au dépôt et à la diffusion de documents scientifiques de niveau recherche, publiés ou non, émanant des établissements d'enseignement et de recherche français ou étrangers, des laboratoires publics ou privés. 


\section{High energy long-lived excited states in DNA double strands}

Ignacio Vayá, François-Alexandre Miannay, Thomas Gustavsson, and Dimitra Markovitsi* ${ }^{*}$

* Dr I. Vayá, F.-A. Miannay, Dr T. Gustavsson, Dr. D. Markovitsi

Laboratoire Francis Perrin

CEA/DSM/IRAMIS/SPAM - CNRS URA 2453

CEA/Saclay

91191 Gif-sur-Yvette, France

Email : dimitra.markovitsi@cea.fr

** This work was supported by the French Agency for Research; project number:

PCV07_194999. We thank Dr Richard Lavery, Dr Krystyna Zakrzewska and Dr Irène Burghardt for helpful discussions. 
The knowledge that UV photons absorbed by DNA induce photoreactions leading to carcinogenic mutations has triggered numerous studies. During the past few years spectroscopic investigations performed with femtosecond resolution brought new insight in the electronic excited states of DNA duplexes. The detailed nature of these states is still under debate $^{[1-6]}$. However, based on results from transient absorption measurements performed for short helices at selected wavelengths, the following picture has been proposed ${ }^{[6-8]}$. The initially populated $\pi \pi^{*}$ states relax within 100 fs to energetically low-lying excimers or exciplexes decaying with time constants up to $c a$. 100 ps. Such partial dissipation of the excitation energy, considered to contribute to the stability of the genetic code ${ }^{[6]}$, was inferred from the steady-state fluorescence spectra of some DNA helices presenting an emission band at longer wavelengths compared to those the monomeric chromophores ${ }^{[5,9,10]}$. However, while monitoring non-emitting transient species, transient absorption measurements do not provide any information about their energy relative to the ground state.

In parallel, theoretical studies pointed out the importance of conformational dynamics on the electronic states of double helices ${ }^{[11,12]}$. Conformational changes affect both the electronic coupling, which determines the properties of exciton states, and the polarity of the local environment, which determines the energy of highly polarizable states. Consequently, the ordering of the various excited states of a duplex may vary with the conformation leading to intricate relaxation pathways. Here we present novel and unexpected fluorescence properties evidenced for duplexes with alternating $\mathrm{GC}$ sequence in their $\mathrm{B}$ form (GC duplexes) which could be explained by such conformational variations. Our observations relate to excited states which emit at energies $c a .4000 \mathrm{~cm}^{-1}$ higher than the $\pi \pi^{*}$ states and decay on the nanosecond time-scale indicating trapping of the excitation energy by high energy channels.

Previous fluorescence upconversion (FU) measurements, which detect only emission from bright excited states, showed that the $\pi \pi^{*}$ states of GC duplexes have a lifetime of $0.2 \mathrm{ps}{ }^{[2}$, ${ }^{13]}$. In the present work, we study the polymer poly(dGdC).poly(dGdC) (ca. $\left.1000 \mathrm{bp}\right)$ and the oligomer $(\mathrm{dGdC})_{10} \cdot(\mathrm{dGdC})_{10}$ by time-correlated single photon counting (TCSPC) using the same laser (100 fs, $267 \mathrm{~nm})$ as for the FU experiments ${ }^{[13]}$. TCSPC also probe emission from "dark" states which may borrow oscillator strength from close-lying bright states. The timeresolved signals are correlated with the steady-state fluorescence spectra. Such a parallel study, in connection with the femtosecond investigation, is necessary in order to disentangle 
complex processes. Probably for this reason, the fluorescence spectrum of poly(dGdC).poly(dGdC), reported about two decades ago, did not reveal the full picture ${ }^{[14]}$. A key point in our experiments was the use of low excitation intensities $\left(<3 \mathrm{~kW} / \mathrm{cm}^{2}\right.$ peak power) in order to avoid photodamage of the duplexes.

The fluorescence spectra of GC duplexes are presented in Figure 1a. The polymer spectrum is characterized by a sharp peak close to the Raman line. The oligomer spectrum is about three times less intense than that of the polymer and has a rather flat profile. Despite the difference in spectral shape, the decays of both duplexes at $305 \mathrm{~nm}$ (Figure 1b) contain a common feature: a long component decaying on the nanosecond timescale. An ultrafast component, which cannot be resolved by TCSPC, is also present in the decays, its contribution being more important in the case of the oligomer.

The amplitude of the fast component becomes larger when the emission wavelength increases from 305 to $330 \mathrm{~nm}$ (Figure 2). The latter wavelength corresponds to the maximum of the spectrum recorded at $200 \mathrm{fs}^{[13]}$. The details of the fits with multi-exponential functions are given in the supporting information. The most important outcome is that the major contribution to the fluorescence decays of both the polymer and the oligomer is related to a time constant of $1.31 \pm 0.05 \mathrm{~ns}$. The associated fluorescence anisotropy (insets in Figure 2) is also independent of the duplex size. After $100 \mathrm{ps,} \mathrm{when} \mathrm{the} \mathrm{instrumental} \mathrm{response} \mathrm{function}$ becomes negligible, the fluorescence anisotropy is close to zero, suggesting that the longlived emission arises from internal conversion and/or an energy transfer process.

The common behavior of the long-lived emission between polymer and oligomer shows that this is not related to an impurity because these compounds were prepared by diffent methods: the polymer biochemically (Amersham Biosciences) and the oligomer by chemical synthesis and annealing of the two parent strands (Eurogentec). One could also think that florescence from an impurity present in the buffer (which does not gives such signals by itself) could be enhanced by intercalation into the duplexes. Yet, the same peak around 300 $\mathrm{nm}$ is observed when the polymer is dissolved in aqueous solutions containing different salts with the same ionic strength: only $\mathrm{NaCl}$ or $\mathrm{KCl}$ or $\mathrm{NaH}_{2} \mathrm{PO}_{4}$ and $\mathrm{Na}_{2} \mathrm{HPO}_{4}$. Therefore, we conclude that this fluorescence is an intrinsic property of the DNA duplexes.

The steady-state absorption and fluorescence spectra of poly(dGdC).poly $(\mathrm{dGdC})$ are shown in Figure 3 on a wavenumber scale together with the fitted FU spectrum recorded at 0.2 ps. The spectra of an equimolar mixture of the 2'-deoxyguanosine monophosphate (dGMP) and 2'-deoxoctosine monophosphate (dCMP) are also given; their fluorescence lifetimes, related to $\pi \pi^{*}$ states are 0.34 and $0.45 \mathrm{ps}$, respectively ${ }^{[15]}$. The polymer 
fluorescence spectrum, after subtraction of the Raman line and correction for reabsorption of the fluorescence, peaks at $34,000 \mathrm{~cm}^{-1}$ whereas that of non-interacting nucleotides at 30,000 $\mathrm{cm}^{-1}$. The latter value coincides with the maximum of the FU spectrum. A striking feature of the high energy emission band is that is much narrower $\left(2700 \mathrm{~cm}^{-1}\right)$ compared to the low energy one $\left(5000 \mathrm{~cm}^{-1}\right)$. The absorption spectrum of the duplex differs from that of noninteracting nucleotides because the electronic coupling induces delocalization of the excitation over a few bases giving rise to an exciton band ${ }^{[11]}$. This is also attested by energy transfer occurring on the femtosecond time-scale ${ }^{[13]}$.

In order to correlate the above energetic considerations with the fluorescence decays determined for GC duplexes on a time-scale varying over six orders of magnitude, we propose the following scheme, illustrated in Figure 4, where two extreme situations are represented. For highly ordered conformations, efficient intraband scattering leads to a low-lying dark state from which detrapping is prevented by a high barrier. In the case less ordered conformations, localization of the excitation on a single base competes with intraband scattering; as a result we observe $\pi \pi^{*}$ monomer like emission.

Within this scheme, the larger contribution of the long-lived component in the polymer emission compared to the oligomer is explained by the reduction of conformational disorder upon increasing the duplex size ${ }^{[16]}$. Consequently, the collective behavior of the excited states, governed by the electronic coupling, is enhanced. Such a conclusion was deduced from the photophysical properties of oligomers and polymers composed of adenine-thymine pairs $[17,18]$. The size effect is reflected in the CD spectra of GC duplexes reported in the literature: though the spectra both polymeric and monomeric duplexes present the features of the $\mathrm{B}$ form, for the poly $(\mathrm{dGdC})$. poly $(\mathrm{dGdC})$, the negative peak at $253 \mathrm{~nm}$ is $3.4 \pm 0.1$ times more intense than the positive peak at $273^{[19,20]}$ but their intensity ratio amounts to only 2.7 for $(\mathrm{dGdC})_{9} \cdot(\mathrm{dGdC})_{9}{ }^{[8]}$. This comparison clearly shows that the polymeric duplex is characterized by increased order compared with the oligomeric one for which fraying at the ends may occur.

Although experiments with other base sequences are necessary to elucidate the precise nature of the "dark" emitting states of GC duplexes we can consider here after three possibilities: Frenkel excitons, $\mathrm{n} \pi^{*}$ states and charge transfer states. The relatively narrow emission band located at higher energy with respect to the monomer emission recalls the behavior of $\mathbf{J}$ aggregates ${ }^{[21,22]}$. One could imagine that excitons with partial $\mathrm{J}$-aggregate character are formed for example between neighboring guanines or cytosines located on 
complementary strands (Figure 8 in ref. ${ }^{[11]}$ ). Moreover, emission from $\mathrm{n} \pi^{*}$ states of some organic molecules in non-polar media is described in the literature ${ }^{[23]}$. The $n \pi^{*}$ states of monomeric guanine and cytosine lie close to the first $\pi \pi^{*}$ in gas phase whereas in aqueous solutions they are strongly destabilized ${ }^{[24-26]}$. A double helix represents an intermediate situation. A recent time-resolved infra-red study reported that the cytosine $n \pi^{*}$ state in poly $(\mathrm{dGdC})$.poly $(\mathrm{dGdC})$ decays with a time-constant of $31 \mathrm{ps}^{[3]}$. These authors remarked that $4 \%$ of the signals persisted after $1 \mathrm{~ns}$, which could correspond to the $\mathrm{n} \pi^{*}$ of guanosine. Finally, theoretical calculations have described the existence of a variety of charge transfer states in DNA duplexes ${ }^{[12,27,28]}$, both intra- and inter-stand. It would not be surprising if such states, depending on orbital overlap, exhibit weak emission which is very sensitive to conformational disorder.

Theoretical calculations taking into account conformational disorder are blatantly needed in order to bring the "dark" emitting states into the spotlight. However, even the nature of the observed high energy long-lived excited states is not yet determined and their population remains unknown, they are expected to play a role in the photoreactivity of the duplexes. Examination of their involvement in the reaction pathways could improve our understanding of DNA damage.

Keywords: DNA fluorescence - Time-resolved spectroscopy · Excited state relaxation · Excitons · Photochemistry 


\section{REFERENCES}

[1] D. Markovitsi, F. Talbot, T. Gustavsson, D. Onidas, E. Lazzarotto, S. Marguet, Nature 2006, 441, E7.

[2] N. K. Schwalb, F. Temps, Science 2008, 322, 243.

[3] G. W. Doorley, D. A. McGovern, M. W. George, M. Towrie, A. W. Parker, J. M. Kelly, S. J. Quinn, Ang. Chem.-Intern. Ed. 2009, 48, 123.

[4] T. Fiebig, J. Phys. Chem. B 2009, 113, 9348.

[5] W. M. Kwok, C. S. Ma, D. L. Phillips, J. Phys. Chem. B 2009, 113, 11527.

[6] C. T. Middelton, K. de La Harpe, C. Su, U. K. Law, C. E. Crespo-Hernàndez, B. Kohler, Ann. Rev. Phys. Chem. 2009, 60, 13.

[7] C. E. Crespo-Hernández, B. Cohen, B. Kohler, Nature 2005, 436, 1141.

[8] K. de La Harpe, C. E. Crespo-Hernàndez, B. Kohler, ChemPhysChem 2009, 10, 1421.

[9] J. Cadet, P. Vigny, in Bioorganic Photochemistry (Ed.: H. Morrison), John Wiley \& Sons, New York, 1990, pp. 1.

[10] D. Markovitsi, T. Gustavsson, F. Talbot, Photochem. \& Photobiol. Sci. 2007, 717

[11] E. Emanuele, K. Zakrzewska, D. Markovitsi, R. Lavery, P. Millie, J. Phys. Chem. B 2005, 109, 16109.

[12] E. B. Starikov, G. Cuniberti, S. Tanaka, J. Phys. Chem. B 2009, 113, 10428.

[13] F. A. Miannay, A. Banyasz, T. Gustavsson, D. Markovitsi, J. Am. Chem. Soc. 2007, 129, 14574.

[14] C.-R. Huang, S. Georghiou, Photochem. Photobiol. 1992, 56, 95.

[15] D. Onidas, D. Markovitsi, S. Marguet, A. Sharonov, T. Gustavsson, J. Phys. Chem. B 2002, 106, 11367.

[16] X. Zuo, G. Cui, K. M. Merz Jr., L. Zhang, F. D. Lewis, D. M. Tiede, Proc. Natl. Acad. Sci. 2006, 103, 3534.

[17] D. Onidas, T. Gustavsson, E. Lazzarotto, D. Markovitsi, J. Phys. Chem. B 2007, 111, 9644.

[18] D. Onidas, T. Gustavsson, E. Lazzarotto, D. Markovitsi, Phys. Chem. Chem. Phys. 2007, 9, 5143.

[19] R. T. Simpson, H. Shindo, Nucleic Acids Res. 1980, 8, 2093.

[20] G. M. J. SegersNolten, N. M. Sijtsema, C. Otto, Biochemistry 1997, 36, 13241.

[21] H. Fidder, J. Knoester, D. A. Wiersma, J. Chem. Phys. 1991, 95, 7880.

[22] H. Lin, R. Camacho, Y. Tian, T. E. Kaiser, F. Würthner, I. G. Scheblykin, Nano Lett. 2010, DOI: $10.1021 / \mathrm{n} 19036559$.

[23] W. M. Nau, U. Pischel, in Organic photochemistry and photophysics (Eds.: V. Ramamurthy, K. Schanze), Taylor \& Francis Group, Boca Raton, London, New York, pp. 75.

[24] L. Serrano-Andres, M. Merchan, A. C. Borin, J. Am. Chem. Soc. 2008, 130, 2473.

[25] L. Blancafort, A. Migani, J. Photochem. Photobiol. A: Chem. 2007, 190, 283.

[26] V. Karunakaran, K. Kleinermanns, R. Improta, S. A. Kovalenko, J. Am. Chem. Soc. 2009, 131, 5839.

[27] F. Santoro, V. Barone, R. Improta, ChemPhysChem 2008, 9, 2531.

[28] A. W. Lange, J. M. Herbert, J. Am. Chem. Soc. 2009, 131, 3913. 



Figure 1. Steady-state fluorescence spectra (a) and fluorescence decays at $305 \mathrm{~nm}$ (b) of poly $(\mathrm{dGdC}) \cdot$ poly $(\mathrm{dGdC})$ (red) and $(\mathrm{dGdC})_{10} \cdot(\mathrm{dGdC})_{10}$ (blue) in phosphate buffer $(0.1 \mathrm{M}$ $\mathrm{NaH}_{2} \mathrm{PO}_{4}, 0.1 \mathrm{M} \mathrm{Na}_{2} \mathrm{HPO}_{4}$ and $0.25 \mathrm{M} \mathrm{NaCl}$ ) following excitation at $267 \mathrm{~nm}$. The intensity of the spectra is representative of the relative quantum yields; the TMP spectrum (fluorescence quantum yield: $1.5 \times 10^{-4}$ ) is shown in black (a). The instrumental response function of the TCSPC (Raman line at $294 \mathrm{~nm}$ ) is shown in grey (b); time-resolution after deconvolution: 10 ps. 

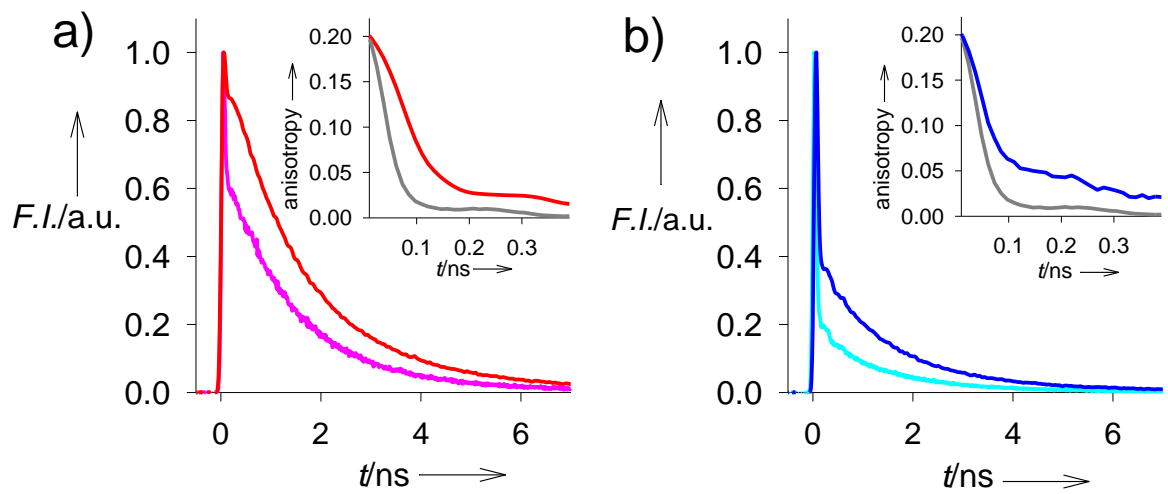

Figure 2. Fluorescence decays obtained for (a) poly(dGdC).poly $(\mathrm{dGdC})$ and (b) $(\mathrm{dGdC})_{10} \cdot(\mathrm{dGdC})_{10}$ at $305 \mathrm{~nm}$ (red and blue) and $330 \mathrm{~nm}$ (pink and cyan) in phosphate buffer $\left(0.1 \mathrm{M} \mathrm{NaH}_{2} \mathrm{PO}_{4}, 0.1 \mathrm{M} \mathrm{Na}_{2} \mathrm{HPO}_{4}\right.$ and $\left.0.25 \mathrm{M} \mathrm{NaCl}\right)$. Insets: fluorescence anisotropy decays at $305 \mathrm{~nm}$; the instrumental response function, normalized to the maximum anisotropy, is shown in grey. Excitation wavelength: $267 \mathrm{~nm}$. 

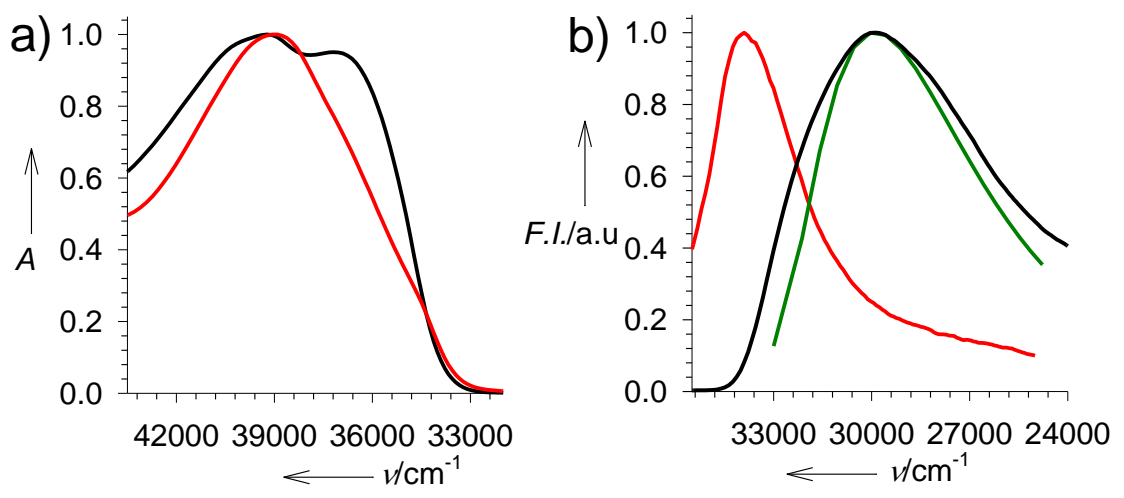

Figure 3. Normalized absorption (a) and fluorescence (b) spectra obtained for an equimolar mixture of non-interacting monomeric chromophores, dGMP and dCMP (black) and poly $(\mathrm{dGdC})$.poly $(\mathrm{dGdC})$ (red). The spectrum in green corresponds to the fit of the fluorescence upconversion spectrum recorded for the polymer at $0.2 \mathrm{ps}^{[13]}$ by a log-normal function. 


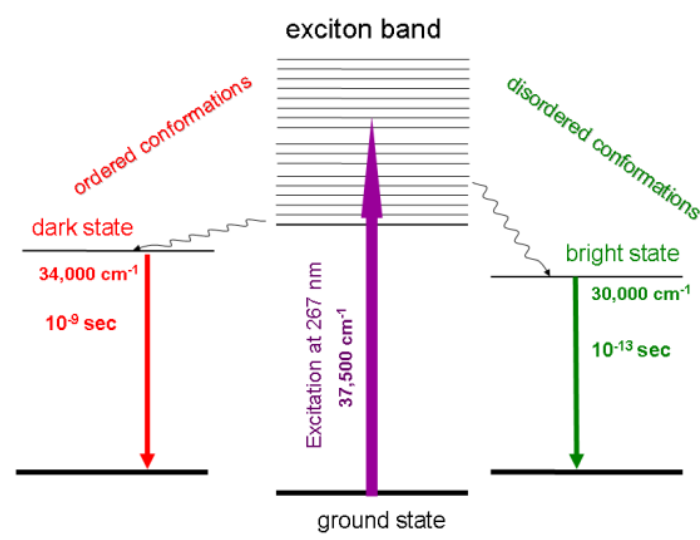

Figure 4. Simplified scheme illustrating emission of GC duplexes either from dark or from bright excited states. For highly ordered conformations, efficient intraband scattering leads to a low-lying dark state from which detrapping is prevented by a high barrier. In the case of less ordered conformations, localization of the excitation on a single base may take place; as a result we observe monomer like emission from a bright $\pi \pi^{*}$. 


\section{TOC}

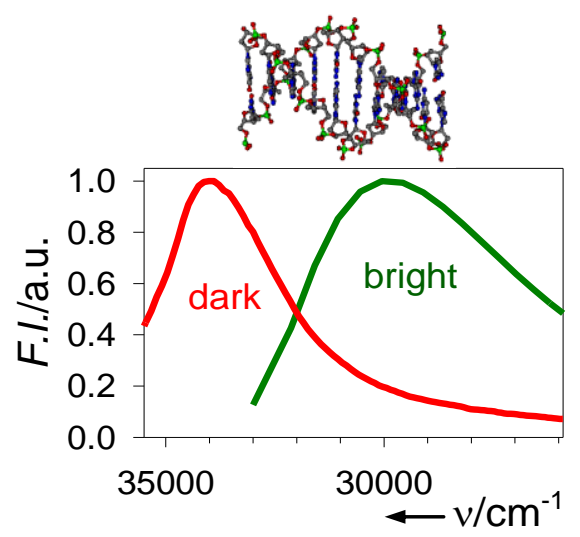

Dark DNA light: “dark" excited states of alternating GC double strands emit fluorescence at $4000 \mathrm{~cm}^{-1}$ higher energy and with four orders of magnitude longer lifetime compared to the bright $\pi \pi^{*}$ states. Such high energy long-lived excited states are expected to play a key role in the DNA photoreactivity associated with the appearance of carcinogenic mutations. 


\section{Supporting Information}

\section{Materials}

The polymer poly $(\mathrm{dGdC})$.poly $(\mathrm{dGdC})$ was purchased from Amersham-Bioscience and delivered as powder which was dissolved in the phosphate buffer $\left(0.1 \mathrm{M} \mathrm{NaH}_{2} \mathrm{PO}_{4}, 0.1 \mathrm{M}\right.$ $\mathrm{Na}_{2} \mathrm{HPO}_{4}$ and $0.25 \mathrm{M} \mathrm{NaCl}$ ). Mild heating to $50^{\circ} \mathrm{C}$, which could destroy possible higher order structures, did not change the photophysical properties. The oligomer $(\mathrm{dGdC})_{10} \cdot(\mathrm{dGdC})_{10}$ was provided by Eurogentec after purification by polyacrylamide gel electrophoresis in the same buffer. More than ten batches of each type of duplex were tested. Water was delivered by a MILLIPORE (Milli-Q Synthesis) system.

\section{Experimental setups and methods}

Steady-state spectra were obtained with a Perkin-Elmer Lambda 900 spectrophotometer and two SPEX spectrofluorimeters (Fluorolog-2 and Fluorolog-3). Emission spectra were recorded at a right angle configuration and were corrected for the response of the detection system. The steady-state fluorescence spectra recorded for dilute solutions of GC duplexes (absorbance $<0.5$ over $10 \mathrm{~mm}$ ) appear as a long tail of the intense Raman line of water (294 $\mathrm{nm})$. Therefore, we present in Figure 1a spectra obtained for concentrated solutions (absorbance at $267 \mathrm{~nm}: 1.0 \pm 0.1$ over $2 \mathrm{~mm}$ ). In this case, the Raman line is weak and its contribution can be easily subtracted from the fluorescence spectra. In order to detect fluorescence spectra from concentrated solutions devoid of inner filter effect and with a minimum reabsorption, a 2 x $10 \mathrm{~mm}$ quartz cell was mounted on a holder whose position could be adjusted parallel and perpendicular to the exciting beam. Thus, the spectra of various solutions having the same absorbance at $267 \mathrm{~nm}$ were representative of the fluorescence quantum yield. The general procedure for recording emission spectra from DNA components over a large spectral domain is described in detail elsewhere ${ }^{[15]}$.

The TCSPC setup used the third harmonic of a titanium-sapphire laser at a repetition rate of $4.75 \mathrm{MHz}$. The average laser power $(0.3 \mathrm{~mW})$ was measured with a Melles Griot broadband powermeter. The irradiated area on the surface of the cell was ca. $0.2 \mathrm{~cm}^{2}$. Solutions (typically $3 \mathrm{ml}$ with an absorbance of 0.5 at 267) were contained in a $10 \mathrm{~mm}$ x $10 \mathrm{~mm}$ quartz cell and continuously stirred. The number of photons absorbed during the recording of a decay the measurement was $c a$. $1.6 \times 10^{-8}$ einstein. Successive measurements gave identical decays (Fig. SI-1) which were eventually merged to decrease the signal to noise ratio. We judge that the reproducibility of successive signals recorded for the same solution is a better criterion than 
changes in the absorbance of the sample (Fig. SI-2), used in transient absorption measurements of similar systems ${ }^{[8]}$.

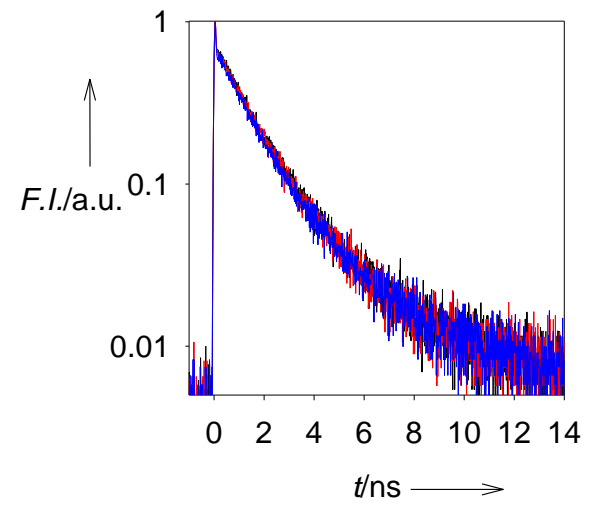

Figure SI-1. Successive fluorescence decays obtained for poly(dGdC).poly(dGdC) at $305 \mathrm{~nm}$ by TCSPC

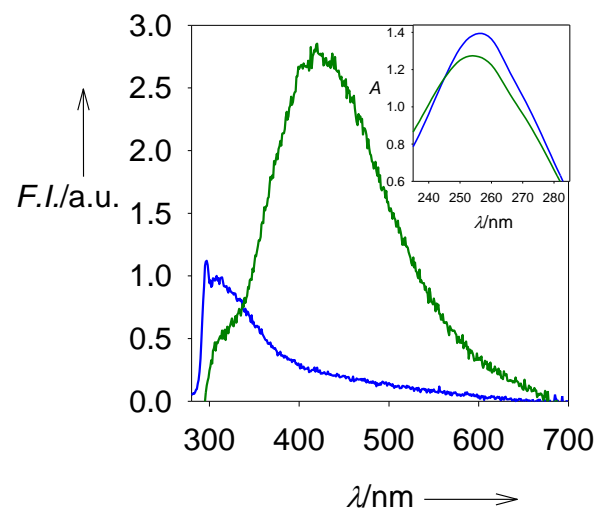

Figure SI-2. Steady-state fluorescence spectra recorded for $(\mathrm{dGdC})_{10} \cdot(\mathrm{dGdC})_{10}$ upon excitation at 267 $\mathrm{nm}$, before (blue) and after (green) irradiation with $267 \mathrm{~nm}$ laser pulses having an intensity 0.5 $\mathrm{GW} / \mathrm{cm}^{2}$. The inset shows the absorption spectra before and after irradiation.

The polarization on the excitation side was controlled by a half-wave plate whereas that of the emission side was fixed vertically by a Glan-Thomson prism. The instrumental response function (50 ps, fwhm) was given by the Raman line of water.

\section{Fits of the fluorescence decays}

We fitted the decays with three-exponential functions $\sum_{i=1}^{3} a_{i} \exp \left(-t / \tau_{i}\right)$ by assigning to $\tau_{1}$ the value of $0.2 \mathrm{ps}$, derived from FU measurements. The results of the fits, together with the 
weights $\left(p_{i}=\alpha_{i} \tau_{1} /\left(\alpha_{1} \tau_{1}+\alpha_{2} \tau_{2}+\alpha_{3} \tau_{3}\right)\right.$ corresponding to each time constant $\tau_{\mathrm{i}}$, are given on Table 1.

Table 1: Parameters derived from the fit of the fluorescence decays of GC duplexes.

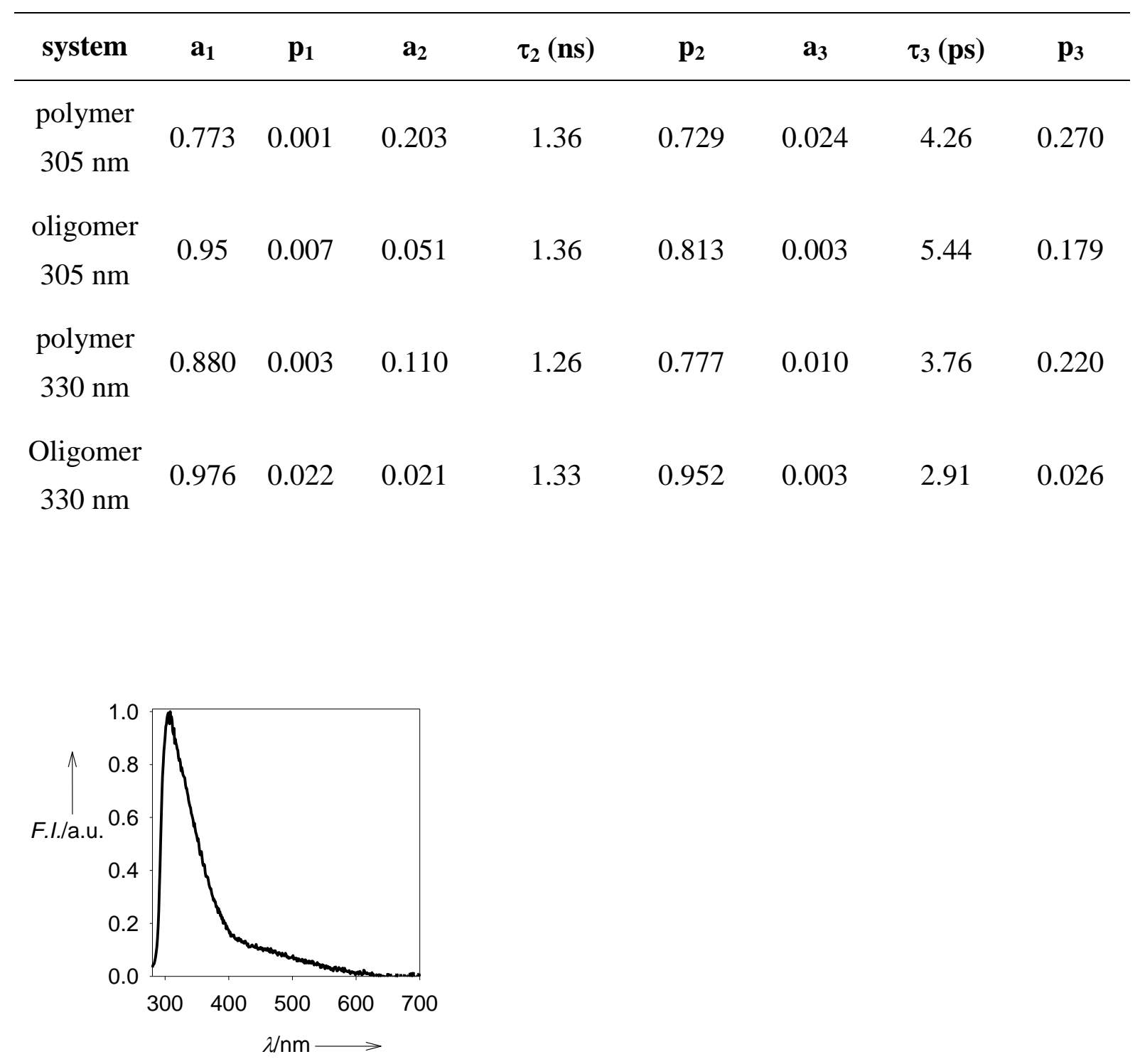

Figure SI 3. Fluorescence spectrum of poly $(\mathrm{dGdC})$.poly $(\mathrm{dGdC})$ in aqueous solution containing $0.375 \mathrm{M} \mathrm{KCl}$. 